\title{
CHARACTERIZATION OF THE FRACTURE MECHANICAL BEHAVIOR OF C-SMC MATERIALS
}

\author{
Martin Tiefenthaler $^{a, *}$, Philipp S. Stelzer $^{a}$, Chi N. Chung ${ }^{a}$, \\ VOLKer REISECKER ${ }^{b}$, ZOLTAN MAJOR ${ }^{a}$ \\ ${ }^{a}$ Institute of Polymer Product Engineering, Johannes Kepler Universität, Altenbergerstraße 69, Linz, Austria \\ ${ }^{b}$ Transfercenter für Kunststofftechnik GmbH, Wels, Austria \\ * corresponding author: martin.tiefenthaler@jku.at
}

Abstract. The fracture mechanics of random discontinuous Carbon Fiber Sheet Molding Compound (C-SMC) materials compared to traditional carbon fiber composites are not well understood. An experimental study was carried out to characterize the fracture behavior of such C-SMC materials. Mode I tests, using double cantilever beam specimens, and mode II tests, adopting the four-point bend, end-notched flexure configuration, were performed. Results show high variations in the forcedeflection responses and scatter in the fracture toughness properties $\mathrm{G}_{\text {Ic }}$ and $\mathrm{G}_{\text {IIc }}$, due to the complex mesostructure defined by random oriented carbon fiber chips. To investigate the influence of the mesostructure, tensile tests with varying specimen width and thickness were assessed by stochastic measures to find the representative specimen size.

Keywords: C-SMC, Carbon Fiber Composites, Fracture Mechanics, Anisotropy.

\section{INTRODUCTION}

The exceptional strength to weight ratios presented by many advanced composite materials are desirable for industries (automotive, aerospace, ... ) that constantly fight to improve efficiency [1]. Random discontinuous C-SMC is a special type of SMC material, where a unidirectional carbon fiber prepreg is slit and chopped into chips, randomly distributed in a mold and finished in a single-stage compression and curing process. This material and process technology can be used to produce geometric complex, lightweight applications in an industrial (automated) and cost efficient way. The composition of the C-SMC material leads to a complex mesostructure, due to the random distribution and discontinuous nature of the chips. Research employing microscopical analysis of tensile, compressive and flexural tested specimens conducted by Feraboli et al. 2] showed that failure in random discontinuous C-SMC materials is a matrixdominated event with two prevalent failure modes of intralaminar chip cracking and interlaminar chip delamination, with little or no fiber breakage. Further resin burn-off and de-plying of the tested specimens suggest that chip delamination is the main cause of structural failure. Also Nicoletto et al. 33 identified that final failure rather occurs due to chip debonding, than due to fiber fracture. The authors point out that delamination failure initiates in chips perpendicular to the load direction and propagates until delamination of other chips with higher alignment is involved. In another recently published work by Kravchenko et al. 4 the tensile failure behavior of discontinuous C-SMC with unidirectional aligned chips was investigated by numerical analysis. A critical length-to-thickness ratio of the chips governing the failure mode was identified, above which chip tensile failure is observed due to the efficient load transfer between the chips. For smaller length-to-thickness ratios chip delamination is prevalent. However, in random discontinuous CSMC materials, the effective load transfer between the chips is greatly reduced because of a wide distribution of overlapping lengths caused by the stochastic distribution of the chips.

Consequently, the main weakness of random discontinuous C-SMC materials was considered to be the interface between the matrix and the fiber chips. Therefore, fracture mechanical tests of mode I and II were carried out to characterize the fracture toughness of this material system and to study the failure behavior in the interface between the chips.

In Figure 11, the inhomogeneous strain field with high scatter is shown on the example of a tensile tested C-SMC specimen analyzed by Digital Image Correlation (DIC).

To get statistical significant values for the macroscopic mechanical behavior and to assess the influence of the mesostructure a experimental Representative Volume Element (RVE) study was conducted. RVE can be defined by:

- the RVE must include a large number of microheterogeneities (inclusions, grains, voids, fibers, etc.) [5];

- The RVE is a model of the material to be used to determine the corresponding effective properties for the homogenised macroscopic model. The RVE should be large enough to contain sufficient information about the microstructure in order to be representative, however it should be much smaller 
than the macroscopic body. (This is known as the Micro-Meso-Macro principle) [5].

- The RVE is defined as the minimum volume of laboratory scale specimen, such that the results obtained from this specimen can still be regarded as representative for a continuum [5].

An experimental study of tensile tests with specimen dimensions varying in width and thickness was performed. Results were analyzed via Oneway-ANOVA.
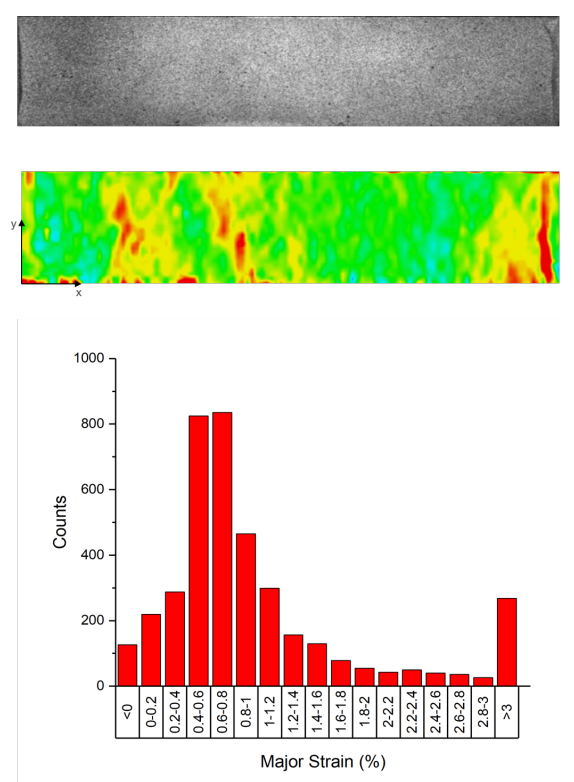

Figure 1. DIC analysis of a tensile test

\section{C-SMC MATERIAL}

The Hexcel prepreg tape (HexMC-i/C/2000/M77 + HexPly M77CS/38\%) consists of high strength carbon fibers with $62 \%$ fiber weight content and the HexPly ${ }^{\circledR}$ M77 resin system [6. HexMC ${ }^{\circledR}$ M77 is produced as shown in Figure 2 the prepreg is slit and chopped into chips $\left(50 \times 8 \mathrm{~mm}^{2}\right)$, randomly distributed and pressed into mats with a width of $460 \mathrm{~mm}$ and a thickness of $2 \mathrm{~mm}$ (uncured) [6]. The material is delivered in semifinished (uncured) rolls which is cut into shape and finished in a (single compression molding) curing step. Plates with dimensions of $350 \mathrm{~mm} \times 350 \mathrm{~mm} \times$ varying thickness (2,4 or 6 layers) for tensile testing (see Figure 4 were produced by Hexcel Holding $\mathrm{GmbH}$, Pasching. Additional plates were manufactured for fracture mechanical tests with dimensions of $245 \mathrm{~mm}$ $\times 245 \mathrm{~mm} \times 2$ Layers thickness containing an adhesive tape, with a width of $50 \mathrm{~mm}$, in the midplane (see Figure 3.

\section{Methodology}

All mechanical tests were performed with the servohydraulic testing machine MTS 852 combined using the Digital Image Correlation (DIC) system ARAMIS, except a few tensile tests (see Table 1) which were

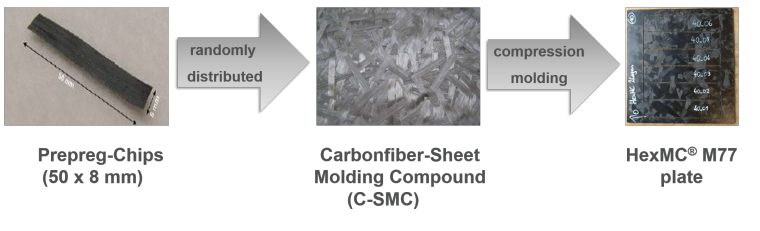

Figure 2. C-SMC production process (reprinted from [6])

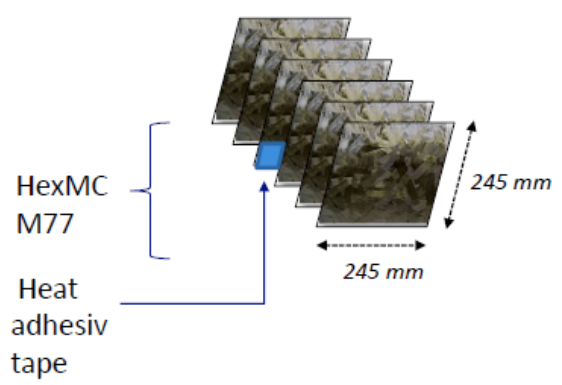

Figure 3. Composition of plate for fracture mechanical tests

carried out with a Zwick/Roell Z150 at TCKT in Wels due to loading limits of the MTS load cell.

\subsection{EXPERIMENTAL RVE STUDY}

In order to assess the RVE size of the used HexMC ${ }^{\circledR}$ M77, an experimental study was carried out: Performed tensile tests with specimen dimensions varying in width and thickness (see Table 1) were statistically analyzed using Oneway ANOVA of the results. ANOVA consists of a comparison of two or more sample groups by testing if their expected values are different and if the variance between the groups is bigger than the variance within a group.

Assumptions of ANOVA:

- Variances of population, treatment and errors are normally distributed

- Error variances are equal between the groups

- Values are independent

The tensile test specimens were milled out of CSMC plates $\left(350 \times 350 \mathrm{~mm}^{2}\right)$ with a diamond coated cutter according to the dimensions (see Figure 4). Specimens were modified through Aluminum tabs $(25$ $\mathrm{mm} \times$ Width $\times 2 \mathrm{~mm}$ ) glued to specimen in grip area in order to prevent failure. Tensile tests were carried out at $1 \mathrm{~mm} / \mathrm{min}$, room temperature and a humidity of $71 \%$. 


\begin{tabular}{lc}
\hline \multicolumn{2}{c}{ Tensile test specimen dimensions } \\
\hline $\begin{array}{c}\text { width } \\
\mathrm{mm}\end{array}$ & $\begin{array}{c}\text { thickness } \\
\mathrm{mm}\end{array}$ \\
\hline $200 \times 15 \times 2 L$ & $200 \times 30 \times 2 L^{*}$ \\
\hline $200 \times 20 \times 2 L$ & $200 \times 30 \times 4 L^{*}$ \\
\hline $200 \times 25 \times 2 L$ & $200 \times 30 \times 6 L^{*}$ \\
\hline $200 \times 30 \times 2 L$ & \\
\hline $200 \times 40 \times 4 L^{*}$ & \\
\hline $200 \times 50 \times 2 L^{*}$ & \\
\hline
\end{tabular}

TABle 1. Tensile test study: varying specimen dimensions; $L=$ Layers; * tests performed on Zwick/Roell Z150

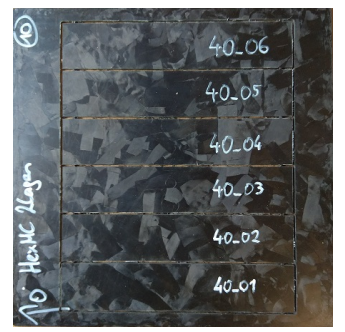

Figure 4. C-SMC plate: milled specimen pattern

\subsection{MODE I}

Mode I tests were performed using five Double Cantilever Beam (DCB) specimens with end blocks $(180 \times 40 \times 6.5$ and an initial crack length of $50 \mathrm{~mm})$. Test setup is shown in Figure 5 Specimens were tested with an unloading/loading procedure according to [7] starting with loading until reaching an axial displacement of $6 \mathrm{~mm}$, where the specimen was first unloaded by $2 \mathrm{~mm}$ and then loaded by $4 \mathrm{~mm}$. These steps where repeated until reaching $20 \mathrm{~mm}$. Mode I tests were carried out at room temperature and at 1 $\mathrm{mm} / \mathrm{min}$ loading rate and at $5 \mathrm{~mm} / \mathrm{min}$ unloading rate [7].

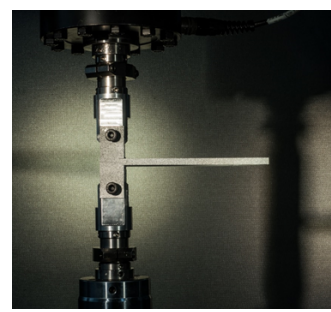

Figure 5. Mode I testing setup

Mode I fracture toughness $\left(\mathrm{G}_{\mathrm{Ic}}\right)$ was calculated via Corrected Beam Theory (CBT) [8]:

$$
G_{I c}=\frac{3 P \delta}{2 B(a+|\Delta|)} \frac{F}{N}
$$

where $P$ is the applied load, $\delta$ the axial displacement, $B$ the specimen width, $F$ the correction factor for large displacement (see Equation 2) and $N$ the correction for loading blocks (see Equation 3). $\Delta$ may be determined experimentally by plotting the cube root of compliance, $C^{\frac{1}{3}}$, as a function of the delamination length $a$ [8].

$$
\begin{gathered}
F=1-\frac{3}{10}\left(\frac{\delta}{a}\right)^{2}-\frac{3}{2}\left(\frac{\delta l_{1}}{a^{2}}\right) \\
N=1-\left(\frac{l_{2}}{a}\right)^{3}-\frac{9}{8} \frac{\delta l_{1}}{a^{2}}\left\{1-\left(\frac{l_{1}}{a}\right)^{2}\right\}-\frac{39}{25}\left(\frac{\delta}{a}\right)^{2} .
\end{gathered}
$$

For the definition of $l_{1}$ and $l_{2}$ see Figure 6

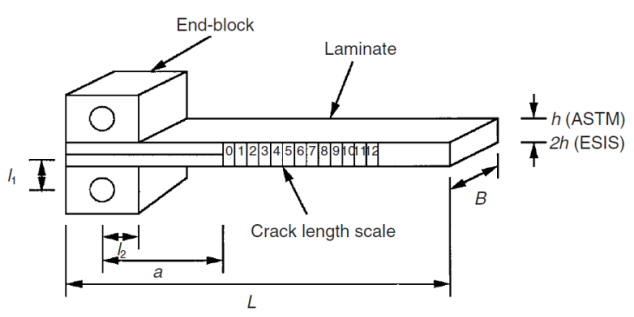

Figure 6. Mode I Double Cantilever Beam (DCB) specimen (reprinted from [8])

\subsection{MODE II}

Mode II tests were performed using five four point bending end notched flexure (4ENF) specimens $(180 \times 40 \times 6.5$ and an initial crack of $50 \mathrm{~mm})$. Monotonic four point bending tests were carried out at room temperature and a loading rate of $1 \mathrm{~mm} / \mathrm{min}$ until failure. Testing setup can be seen in Figure 7. Mode II fracture toughness was calculated via Experimental Compliance Method (ECM) [8]:

$$
G_{I I c}=\frac{3 P^{2} m a^{2}}{2 B},
$$

where $P$ is the applied load, $B$ is the specimen width and $m$ is the slope from the compliance plotted versus the cube delamination length $a^{3}$.

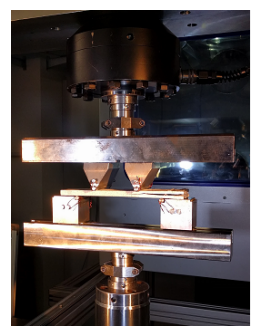

Figure 7. Mode II testing setup

\section{Results And Discussion}

\subsection{EXPERIMENTAL RVE STUDY}

In the Figures 8 and 9, results from the onewayANOVA of the tensile tests are presented, where the black points represent the measured values and the 
green colored diamond shapes display the average mean (horizontal center line), the population size (width of the diamond) and the standard deviation values (height of the diamond). Results from specimens varying in both, width and thickness show high scattering with high standard deviation and error values. No clear trend could be observed by using ANOVA over the experimental window. The scattering of the values was too high and did not show a normal distribution (see right hand side of the Figures 8 and 9, thus it was not possible to determine the RVE by using ANOVA. One reason for the high scattering is the heterogeneous mesostructure due to the in-plane random orientation of the discontinuous chips. The experimental study performed in this work ended at a specimen width of $50 \mathrm{~mm}$, which is also the length of the reinforcing fiber chips. This could be a reason for the high scattering of the values. The results of this study show, that the RVE might be found in larger specimen dimensions.

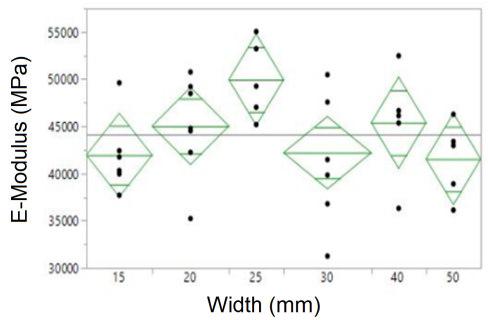

a)

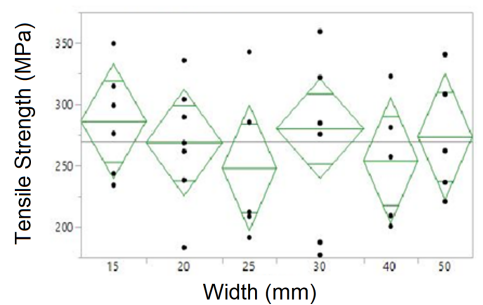

b)

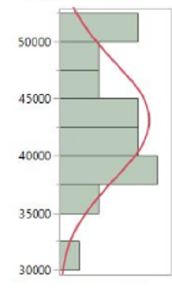

c)

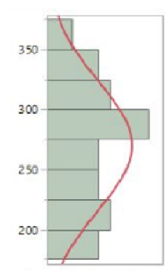

d)
Figure 8. Oneway ANOVA: a) E-Modulus over width, b) Tensile strength over width, c) E-Modulus distribution, d) Tensile strength distribution

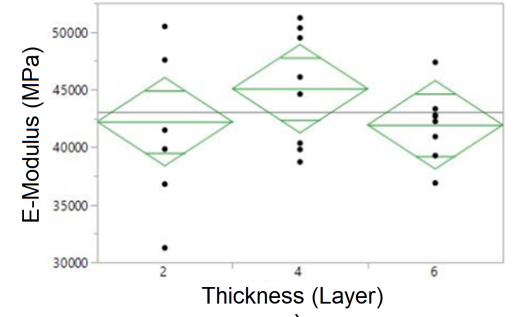

a)

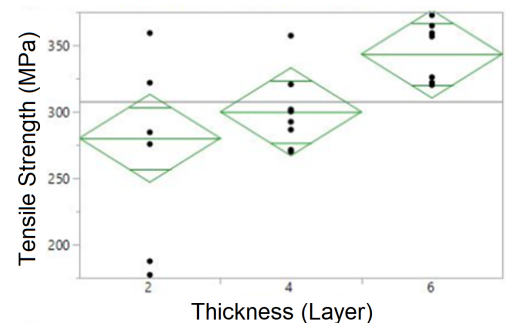

b)

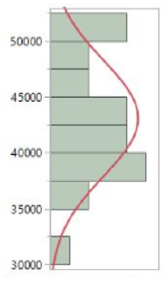

c)

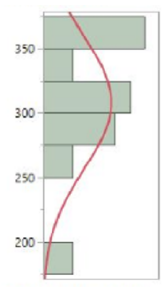

d)
Figure 9. Oneway ANOVA: a) E-Modulus over thickness, b) Tensile strength over thickness, c) E-Modulus distribution, d) Tensile strength distribution

\subsection{MODE I}

In Figure 10, load-displacement curves and mode I fracture toughness are presented. High scattering of $\mathrm{G}_{\text {Ic }}$ values (from 1.53 to $2.69 \mathrm{~mJ} / \mathrm{mm}^{2} ; \mathrm{AVG}=1.83$; $\mathrm{Std}=0.542)$ compared to classical UD laminates was observed.

The load-displacement curves show several small force drops. These drops may occur when fiber chips, carrying the load, fully delaminate. However, load may increase further because the crack stops at the next chips. In C-SMC (Discontinuous Fiber Composite) the initial crack does not correlate to total failure. C-SMC material stop or at least greatly slow crack propagation. However, in Continuous Fiber Composites cracks propagate more easily. Initial failure is visible in load-displacement curves as a sudden load drop and often correlates to ultimate failure [9].

This crack stopping behavior is a result of the random distribution of the discontinuous fiber chips. A similar behavior was also found by Boursier et al. 9] by performing tensile tests with acoustic emission measurements. They found out that first cracks do not correlate with ultimate failure of the tensile specimens which supports further the crack stopping behavior of the C-SMC materials.

\subsection{Mode II}

In Figure 11 load-displacement curves and mode II fracture toughness are presented. High scattering of $\mathrm{G}_{\text {IIc }}$ values (from 2.21 to $3.69 \mathrm{~mJ} / \mathrm{mm}^{2}$; $\mathrm{AVG}=3.13$; Std $=0.551)$ compared to classical UD laminates was observed.

The load-displacement curves of mode II tested specimens show a crack stopping behavior similar to mode I. Values of mode II are less scattering compared 

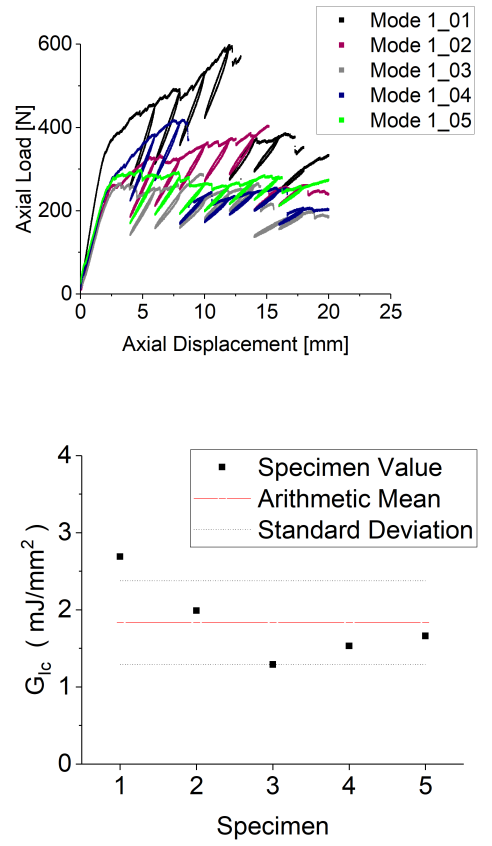

Figure 10. Results: load over displacement (top) and mode I fracture toughnesses comparison (bottom)
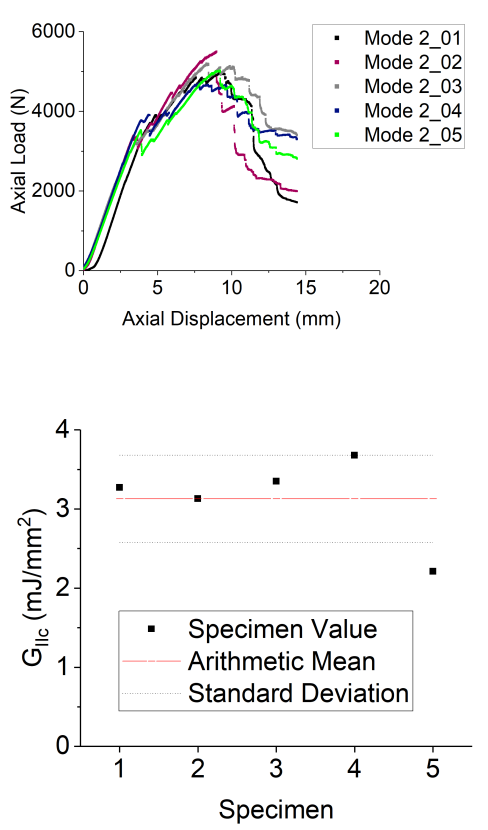

Figure 11. Results: load over displacement (top) and mode II fracture toughnesses comparison (bottom)

to mode I in the linear elastic region (see Figure 11) because specimens are loaded in shear mode.

\section{Conclusion}

Random discontinuous C-SMC is a special type of SMC material, where a unidirectional carbon fiber prepreg is slit and chopped into chips, randomly distributed in a mold and finished in a curing step. The production process leads to a heterogeneous mesostruc- ture with high scattering. A representative specimen size could not be found in the investigated experimental window via ANOVA. However, the conducted experimental RVE study strongly suggests the use of statistical methods to account for the variability of the mechanical properties of C-SMC materials. Nominal values are not significant in the design process and modelling of such materials. The representative size is expected to be at bigger specimen dimensions.

Fracture mechanical test results showed higher scatter of $\mathrm{G}_{\text {Ic }}, \mathrm{G}_{\mathrm{IIc}}$ values of C-SMC material compared to traditional UD laminates. The crack propagation was found not continuous like in traditional UD laminates, but a crack stopping behavior was observed due to the random orientation of the discontinuous chips.

A combination of micromechanical simulations and experimental studies is proposed for a better understanding of the C-SMC fracture behavior, which will be part of future research.

\section{ACKNOWLEDGEMENTS}

This research was performed within the framework of the "0-Waste" project with contributions by Engel GmbH (Schwertberg, AUT) and Alpex Technologies GmbH(Mils, AUT), Hexcel Composites GmbH (Neumarkt, AUT). The "0-Waste" project is funded by the Austrian funding agency FFG. The authors would further like to thank the Transfercenter für Kunststofftechnik GmbH (Wels, AUT) for their contributions.

\section{REFERENCES}

[1] David A. Hawkins Jr., Anwarul Haque. Strain energy release rate and mode-I delamination growth in carbon-graphene/epoxy hybrid nanocomposites 2015 .

[2] Paolo Feraboli, Elof Peitso, Francesco Deleo, Tyler Cleveland. Characterization of prepreg-based discontinuous carbon fiber/epoxy systems 2009.

[3] Gianni Nicoletto, Enrica Riva, Anellino Stocchi. Mechanical characterization of advanced random discontinuous carbon/epoxy composites 2016.

[4] Sergii G. Kravchenko, Drew E. Sommer, R. Byron Pipes. Uniaxial strength of a composite array of overlaid and aligned prepreg platelets 2018.

[5] Inna M. Gitman, Harm Askes, Lambertus J. Sluys, Oriol Lloberas Valls. The concept of representative volume for elastic, hardening and softening materials 2004.

[6] Hexcel. HexMC User Guide.

https://www.hexcel.com/Resources/DataSheets/ HexMC-Molding-Compound, 2014. [Online; accessed 10-May-2018].

[7] Standard Test Method for Mode I Interlaminar Fracture Toughness of Unidirectional Fiber-Reinforced Polymer Matrix Composites, 2001.

[8] John M. Hodgkinson. Mechanical testing of advanced fibre composites. 2000.

[9] Bruno Boursier, Alfonso Lopez. Failure Initiation and Effect of Defects in Structural Discontinuous Fiber Composites 2010. 